\title{
Variations of the Tumor Position in Frameless Lung Sbrt: Assessment of Predictive Factors Including Tumor Volume Changes
}

\author{
Jessica Schuster $^{1 *}$, Michael Myers ${ }^{1}$, Mihaela Rosu ${ }^{1}$, Nitai Mukhopadhyay $^{2}$ and Elisabeth Weiss ${ }^{1}$
}

${ }^{1}$ Department of Radiation Oncology, Virginia Commonwealth University, Richmond, VA, USA

${ }^{2}$ Department of Biostatistics, Virginia Commonwealth University, Richmond, VA, USA

\begin{abstract}
Purpose: To quantify inter- and intrafractional variations of tumor position and analyze the relationship between these changes and respiratory motion amplitude, volume changes and tumor location, in frameless stereotactic body radiotherapy (SBRT) of lung tumors.

Materials and methods: Tumor volumes and bony landmarks were contoured manually by a single physician on 174 pre- and under treatment cone-beam computed tomographies (CBCTs) of 17 patients. The interfraction variation of the tumor position was measured by comparing the centroid position of the tumor relative to bony anatomy of each fraction to the pretreatment CBCT scans. The intrafraction variation was measured by comparing the pretreatment tumor location to under treatment $C B C T$ s for every fraction. Respiratory motion was analyzed on planning 4D fan beam CTs for all patients. The change in tumor volume was determined by comparing the contoured tumor volumes on sequential pretreatment CBCTs.

Results: The average interfraction/intrafraction tumor displacementrelative to bony landmark in $\mathrm{mm}$ was 0.6 (SD 2.3) /-0.3 (SD 0.7) in mediolateral, -0.7 (SD 3.8) /0.0 (SD 2.1) in anteroposterior, and -0.6 (SD 5.9) /-0.2 (SD 2.3) in craniocaudal direction. Inter-/intrafraction tumor-to-bone variations $>3 \mathrm{~mm}$ were observed in $60 \% / 14 \%$ of scans respectively. On the initial $\mathrm{CBCT}$, the average tumor volume was $9 \mathrm{~cm}^{3}\left(\right.$ range $\left.1-37 \mathrm{~cm}^{3}\right)$ with a mean volume reduction over the treatment course of $12 \%$ (range, $+14 \%$ to $-54 \%$ ). Patients with a pretreatment motion amplitude $>9 \mathrm{~mm}(p=0.002)$, peripheral tumor location $(p=0.04)$, and volume change $>12 \%(p=0.009)$ had larger interfraction displacement in lateral direction.

Conclusions: Frameless set up is comparable to patient positioning with more elaborate fixation devices Tumor position variations relative to bony anatomy are an important source of geometric uncertainty providing a rationale for repeated soft tissue-based image guidance, particularly in patients identified in this study to be at higher risk for variations.
\end{abstract}

Keywords: SBRT; Inter-/Intrafraction position variation; Tumor volume change

\section{Introduction}

Stereotactic body radiation therapy (SBRT) has local control rates for inoperable patients similar to those reported for operable patients after surgical resection and has emerged as the preferred radiotherapy modality in early stage, inoperable NSCLC [1-4]. Highly conformal dose delivery requires accurate knowledge of the target position. In order to achieve the required geometrical precision, SBRT is often performed with complex immobilization systems, such as stereotactic frames, evacuated cushions with or without abdominal compression, and/or cone-beam CT (CBCT) for soft tissue-based patient set up [58]. СВCT has been used to measure inter-/intrafractional positional variations [9-10], to qualitatively and quantitatively identify potential sources of positional variability $[11,12]$, and to determine dosimetric impact of positional variability $[5,13]$. A known source of positional variation for conventional radiotherapy results from tumor regression as demonstrated by several studies showing average tumor regression of about $40 \%$ using $4 \mathrm{D}$-FBCT and CBCT $[14,15]$. Treatment duration is greatly reduced with SBRT technique; therefore, tumor volume changes are currently assumed to be irrelevant. A paper reviewing dosimetric impact of repeated CBCT during lung stereotactic radiation delivery by Galerani et al. [6] found volume change to be minimal.

The purpose of our study was to quantify inter- and intrafractional variation for frameless SBRT delivery to lung tumors by utilizing repeated CBCTs. While standard use of $\mathrm{CBCT}$ allows for tumor-based set up and reduces interfractional uncertainties $[6,16]$, the evaluation of the extent of intrafraction positional variability particularly in frameless patient set up is currently limited. In addition, tumor characteristics such as volume and changes thereof, tumor location and respiratory motion amplitude were analyzed to predict for increased positional variation.

\section{Material and Methods}

\section{Patients}

Between March 2008 and September 2009, 17 sequential patients underwent SBRT for either primary lung cancer or isolated metastatic lung disease. All patients had a tumor size limited to $\leq 5$ $\mathrm{cm}$ in maximum dimension. Fourteen patients had peripheral tumor.

*Corresponding author: Jessica Schuster, MD, Department of Radiation Oncology, Virginia Commonwealth University, 401 College Street, PO Box 980058, Richmond, VA, USA, 23298, Tel: 804-828-9463; Fax: 804-828-6042; E-mail: jschuste@mcvh-vcu.edu

\section{Received April 02, 2013; Accepted April 18, 2013; Published April 21, 2013}

Citation: Schuster J, Myers M, Rosu M, Mukhopadhyay N, Weiss E (2013) Variations of the Tumor Position in Frameless Lung Sbrt: Assessment of Predictive Factors Including Tumor Volume Changes. J Nucl Med Radiat Ther 4: 147. doi:10.4172/2155-9619.1000147

Copyright: (C) 2013 Schuster J, et al. This is an open-access article distributed under the terms of the Creative Commons Attribution License, which permits unrestricted use, distribution, and reproduction in any medium, provided the original author and source are credited. 
Citation: Schuster J, Myers M, Rosu M, Mukhopadhyay N, Weiss E (2013) Variations of the Tumor Position in Frameless Lung Sbrt: Assessment of Predictive Factors Including Tumor Volume Changes. J Nucl Med Radiat Ther 4: 147. doi:10.4172/2155-9619.1000147

Page 2 of 5

Central tumor location was defined as within $3 \mathrm{~cm}$ of heart, aorta, spinal cord, esophagus and main airways. A total dose of 48-60 Gy was delivered in 4-6 fractions prescribed to PTV covering isodose. The approximate treatment time per fraction was 30 minutes; the duration of the treatment course averaged 10 days (range 8-14 days). All patients completed informed consent which had been approved by the local institutional review board.

\section{D CT and CBCT acquisition}

Patients were immobilized in the supine position with both arms above the head using an AccuFix ArmShuttle (Qfix Systems, Avondale, PA, USA). A free breathing four-dimensional computed tomography (4DCT, Brilliance Big Bore, Philips Medical Systems, Andover, MA) simulation procedure was used for all patients $(120 \mathrm{kV}, 400 \mathrm{mAs}$, slice thickness $3 \mathrm{~mm}$ ). The isocenter was marked with three skin tattoos. The scan range covered the complete thoracic region. For each 4DCT, respiration motion was monitored by tracking the vertical displacement of the upper abdominal wall using the Real-time Position Management System (Varian Medical Systems, Palo Alto, CA). The 4DCT was reconstructed with 10 respiratory phases sorted prior to transfer to Pinnacle (Pinnacle 8.1, Phillips, Fitchburg, WI) for treatment planning.

The pretreatment CBCTs (Varian, Medical Systems, Palo Alto, CA) were performed over 1 minute using $80 \mathrm{kV}, 25 \mathrm{mAs}$ following patient alignment to skin tattoos. Treatment was delivered following rigid registration between $\mathrm{CBCT}$ and the time-average phase of the simulation $4 \mathrm{D}-\mathrm{CT}$ as a reference on a Varian Trilogy machine using tumor alignment. For each fraction, CBCT was typically obtained prior to, and after completion of the first and second third of the treatment. Corrections were applied with $3 \mathrm{~mm}$ action threshold.

\section{Target delineation}

The gross tumor volume (GTV) was contoured in preset lung window on each of the 10 respiratory phases of the planning 4D-CT using diagnostic CT scans and PET scans as reference.

GTVs on all CBCTs were contoured in default lung window by one physician using a Pinnacle research version (Pinnacle version 8.1, Philips Medical Systems, Milpitas, CA). For bony landmarks, a vertebral body close to the tumor location was contoured in the default mediastinal window. All contours were then reviewed by another physician for plausibility. In total 174 CBCT scans (range 4-17 CBCTs per patient) including 47 pretreatment scans and 127 intrafraction scans were contoured. Intraobserver contouring variability was evaluated by repeated contouring of 3 patients' lung tumor and reference vertebra on the initial CBCT at three different time points.

\section{Interfraction and intrafraction tumor-to-bone displacement}

To measure the distance between tumor center of mass (centroid) and bony reference structure, vectors between the centroids of both structures were calculated for the mediolateral (ML), anteroposterior (AP), and craniocaudal (CC) directions. Interfraction tumor displacement relative to bony anatomy was calculated as the differences of the tumor-bone vector between the first $\mathrm{CBCTs}$ of each subsequent fraction relative to the first CBCT of the first fraction. Intrafraction tumor displacement was calculated by comparing the vectors obtained on during therapy-CBCT scans to the pre-therapy scan. Displacements were averaged over all scans per fraction, over all fractions and all patients. Positive displacement is represented by motion in the left, inferior, and anterior direction, negative displacement is represented by right, posterior, and superior direction.

\section{Range of tumor motion and volume analysis}

Using the tumor contours of the 10 phases of the planning $4 \mathrm{D}$ $\mathrm{CT}$, the range of motion was calculated as the maximum difference of centroids between all phases for each patient. The percentage tumor volume change of subsequent fractions was calculated relative to the first $\mathrm{CBCT}$ of the first fraction.

Statistical analysis of inter- and intrafractional positional variations in relationship with tumor size, position and volume

Inter- and intrafraction positional variations were analyzed by repeated longitudinal modeling with positional variation in each direction as the response and longitudinal volume change, absolute volume, tumor location (peripheral versus central), and respiratory motion as the covariates. Average positional variation in each direction and average three-dimensional variation were tested for association with the same parameters as above with an ANOVA model. Significance was assumed for $\mathrm{p}<0.05$.

\section{Results}

\section{Contouring variability}

Repeated contouring of tumor and vertebra in 3 patients resulted in highly consistent contours, showing volume variation of less than $3 \%$ for tumors and $1 \%$ for vertebral bodies when structures were recontoured at three different time points without review of previous contours by single physician.

\section{Interfraction tumor-to-bone displacement}

Using first pretreatment CBCT as reference, tumor displacements relative to bone $>3 \mathrm{~mm}$ in at least one direction were observed in $60 \%$ of subsequent fractions. Displacement greater than $3 \mathrm{~mm}$ was most frequent in the craniocaudal followed by anteroposterior direction. The average interfraction tumor displacement vector for all patients relative to bony anatomy was $0.6 \mathrm{~mm}$ (SD $2.3 \mathrm{~mm}$ ), $-0.7 \mathrm{~mm}$ (SD $3.8 \mathrm{~mm}$ ), and $-0.6 \mathrm{~mm}$ (SD $5.9 \mathrm{~mm}$ ) in the ML, AP, and CC directions, respectively.

\section{Intrafraction tumor-to-bone displacement}

Compared to the pretreatment scan of each fraction, tumor displacements relative to bone greater than $3 \mathrm{~mm}$ were appreciated in $14 \%$ of intrafraction scans. Similar to the interfraction data, craniocaudal followed by anteroposterior were the directions with most frequent displacements $>3 \mathrm{~mm}$. The average intrafraction tumor displacement for all patients was $-0.3 \mathrm{~mm}$ (SD $0.7 \mathrm{~mm}), 0.0 \mathrm{~mm}$ (SD $2.1 \mathrm{~mm}$ ), and $-0.2 \mathrm{~mm}$ (SD $2.3 \mathrm{~mm}$ ) in the $\mathrm{ML}, \mathrm{AP}$, and CC directions, respectively. Represented in figure 1 are individual and average intrafraction vector coordinates for fractions 1 through 4 . As there were only three patients with five fractions, the intrafraction from fifth fraction vectors were not included on the graph.

\section{Respiration motion and tumor volume change}

The averagerange of breathing motion of the lung tumors on planning 4D CTs was $9 \mathrm{~mm}$ (range 2-20 mm). On the initial CBCT obtained prior to first fraction delivery, the average tumor volume was $9 \mathrm{~cm}^{3}$ (range 1-37 $\mathrm{cm}^{3}$ ). Volumetric analysis showed tumor shrinkage in 11 of 13 patients with average percent change of $-12 \%$ (range +14 to $-54 \%$ ) over a treatment course (Figure 2). The average changes by fraction with respect to the first fraction were - $4 \%$ for fraction $2,-13 \%$ for fraction $3,-18 \%$ for fraction 4 , and -22.0 for fraction 5 . 
Citation: Schuster J, Myers M, Rosu M, Mukhopadhyay N, Weiss E (2013) Variations of the Tumor Position in Frameless Lung Sbrt: Assessment of Predictive Factors Including Tumor Volume Changes. J Nucl Med Radiat Ther 4: 147. doi:10.4172/2155-9619.1000147

Page 3 of 5

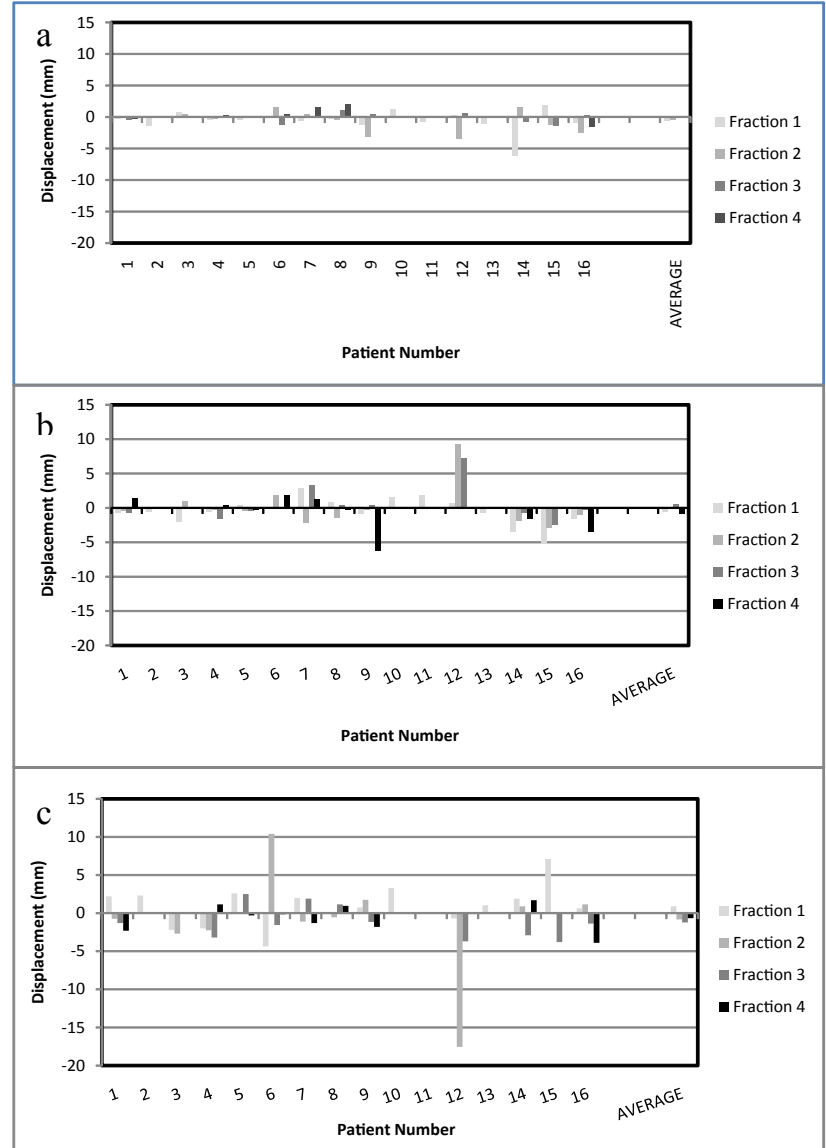

Figure 1: Intrafraction displacement. Intrafraction displacement averages by coordinate direction per fraction relative to first CBCT of each fraction. ML: mediolateral, AP: anteroposterior, CC: craniocaudal.

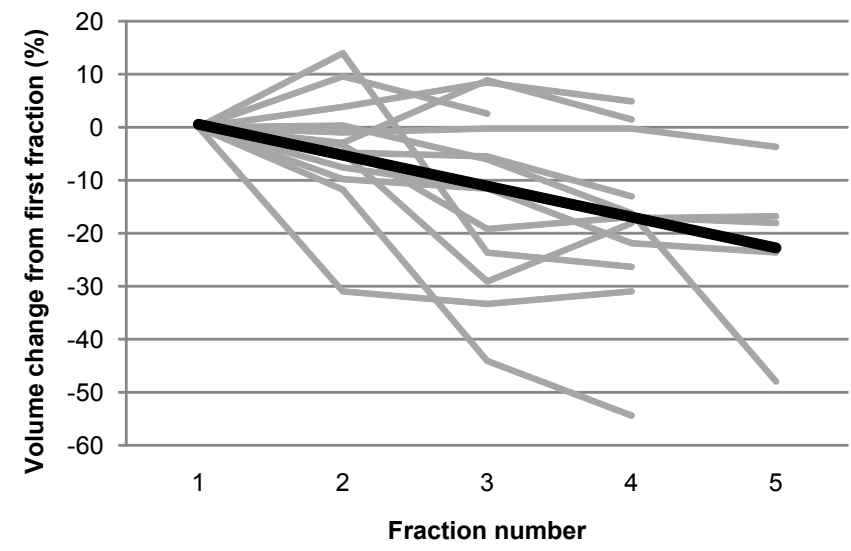

Figure 2: Volumetric analysis of 13 patients. Each line point representing the percent volume change from first CBCT obtained immediately prior to the first fraction. The average tumor volume reduction is represented by the thick line.
Effect of breathing motion, volume change, tumor size and location on inter- and intrafraction positional variation

Interfraction and intrafraction displacement vectors as individual Cartesian coordinates and Euclidian averages were analyzed to determine the relationship with respiratory motion amplitude, initial tumor volume, overall and per fraction tumor volume change, and tumor location. A longitudinal analysis showed interfraction displacement in ML direction was significantly associated with motion amplitude $>$ or $<9 \mathrm{~mm}(\mathrm{p}<0.002)$ (Figure $3 \mathrm{a})$, volume change $>$ or $<12 \%(\mathrm{p}<0.009)$ (Figure $3 \mathrm{~b})$, and tumor position peripheral versus central $(\mathrm{p}<0.04)$. Initial tumor volume, positional variations in AP
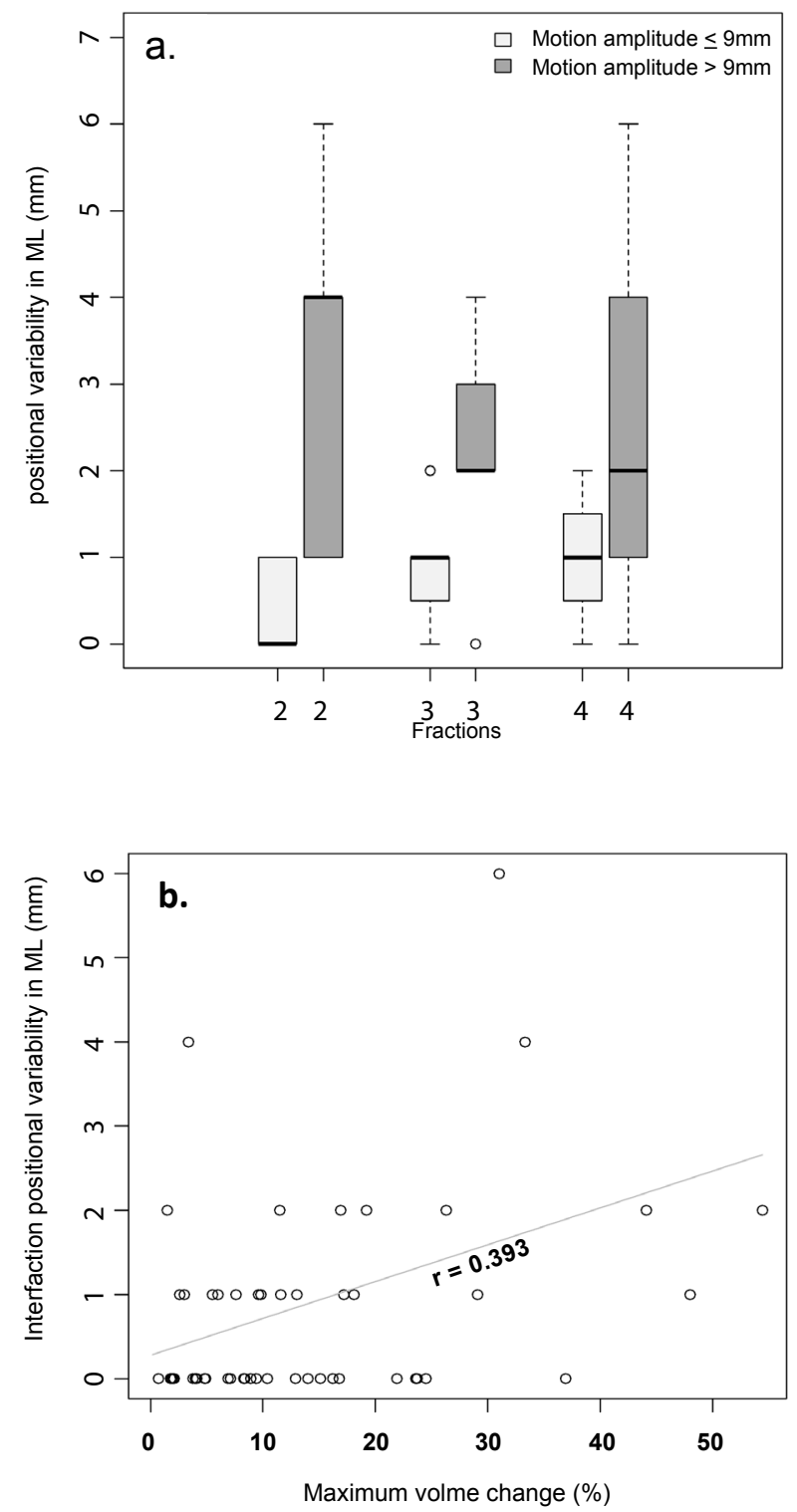

Figure 3: $\mathbf{a}$ and b: Interfraction displacment. Represent increased interfraction displacement in the setting of a) tumor amplitude greater than $9 \mathrm{~mm}$ and $\mathrm{b}$ ) percent volume change per fraction and patient relative to the CBCT of the first fraction. 
Citation: Schuster J, Myers M, Rosu M, Mukhopadhyay N, Weiss E (2013) Variations of the Tumor Position in Frameless Lung Sbrt: Assessment of Predictive Factors Including Tumor Volume Changes. J Nucl Med Radiat Ther 4: 147. doi:10.4172/2155-9619.1000147

Page 4 of 5

\begin{tabular}{|c|c|c|c|c|c|c|c|}
\hline Patient & Age $(y)$ & Motion Amplitude (mm) & Clinical Stage & Histology type & $\begin{array}{l}\begin{array}{l}\text { Tumor location } \\
\text { (lung lobe) }\end{array} \\
\end{array}$ & $\begin{array}{l}\text { Tumor location (peripheral vs. } \\
\text { central) }\end{array}$ & Tumor Volume $\left(\mathrm{cm}^{3}\right)$ \\
\hline 1 & 53 & 3 & T1aNOM0 & SCC & RUL & $P$ & 7.1 \\
\hline 2 & 76 & 5 & T1bNOMO & SCC & RUL & $P$ & 9.4 \\
\hline 3 & 67 & 3 & T1aNOMO & SCC & LUL & $P$ & 4.1 \\
\hline 4 & 66 & 5 & M1 & RCC & LUL & $P$ & 0.7 \\
\hline 5 & 51 & 3 & M1 & NSCLC & RUL & C & 1.9 \\
\hline 6 & 85 & 5 & T1bNOM0 & SCC & RUL & $C$ & 12.9 \\
\hline 7 & 74 & 2 & T2NOMO & SCC & RUL & $\mathrm{P}$ & 36.9 \\
\hline 8 & 66 & 10 & T1aNOMO & NSCLC & RML & $p$ & 1.8 \\
\hline 9 & 68 & 10 & T1aM0M0 & A & LLL & $P$ & 2.0 \\
\hline 10 & 60 & 5 & T1bN0M0 & A & RUL & $\mathrm{P}$ & 6.9 \\
\hline 11 & 88 & 20 & T1aNOMO & A & LLL & $P$ & 15.1 \\
\hline 12 & 63 & 10 & T1aNOMO & A & RLL & $P$ & 4.0 \\
\hline 13 & 69 & 3 & LR & SCC & LUL & $P$ & 10.4 \\
\hline 14 & 83 & 5 & T1bNOMO & A & LUL & $\mathrm{P}$ & 8.3 \\
\hline 15 & 68 & 20 & T1aNOMO & A & RLL & $P$ & 2.1 \\
\hline 16 & 52 & 17 & T1aNOM0 & A & RLL & $\mathrm{P}$ & 2.7 \\
\hline 17 & 69 & 20 & T1bNOMO & A & RLL & C & 24.5 \\
\hline
\end{tabular}

Abbreviations: Y: Years; SCC: Squamous Cell Carcinoma; A: Adenocarcinoma; RCC: Renal Cell Carcinoma; NSCLC: Non Small Cell Lung Cancer- not otherwise specified; RUL: Right Upper Lobe; LUL: Left Upper Lobe; P: Peripheral; C: Central, LR: Local Recurrence

Table 1: Patient and tumor characteristics.

or CC directions and intrafraction variations were not significantly associated with any of the above characteristics (Table 1).

\section{Discussion}

Positional variability was investigated by analysis of sequential CBCTs showing feasibility of frameless, free-breathing set-up and identifying patients at higher risk for tumor positional variability.

\section{Positional variability with frameless patient setup}

Of analyzed CBCT images, $60 \%$ of interfraction scans and $14 \%$ of intrafraction scans had tumor displacements greater than our action threshold of $3 \mathrm{~mm}$ with respect to bony landmark, but all average interfraction/intrafraction tumor displacementsrelative to bony landmarks were all $<3 \mathrm{~mm}$. The largest standard deviation was in craniocaudal direction as expected given this is the axis of greatest respiratory amplitude. Our reported inter/ intrafraction tumor displacements are comparable to previous studies that used stereotactic patient positioning equipment $[11,12]$ and frameless equipment $[11,16,17]$. Guckenberger et al. [12] used stereotactic frame immobilization and demonstrated intrafraction tumor position variation of $0.4 \mathrm{~mm} \mathrm{ML}$ (SD 1.0), $-0.8 \mathrm{~mm} \mathrm{AP} \mathrm{(SD} \mathrm{1.7),} \mathrm{and} 0.7 \mathrm{~mm} \mathrm{CC}$ (SD 1.7). Comparing soft-tissue post-correction, pre-treatment CBCTs and post-treatment CBCTs, Shah et al. [11] observed $0.01 \mathrm{~mm} \mathrm{ML}$ (SD 1.5), $0.1 \mathrm{~mm}$ AP (SD 1.9), and $0.2 \mathrm{~mm} \mathrm{CC} \mathrm{(SD} \mathrm{1.8)} \mathrm{intrafraction}$ displacements with different types of immobilization: stereotactic frame, BodyFIX, or hybrid (alpha cradle+BodyFIX). Utilizing a frameless free-breathing set-up, but $4 \mathrm{D}$ CBCT, Sonke et al. [17] investigated set up accuracy and validated use of small PTV margins during SBRT. 4D CBCT acquisition has so far not been widely available in routine clinical practice. We therefore report our experience with $3 \mathrm{D}$ CBCT. Additional key differences are use of a combination of frameless immobilization, free breathing scans CBCTs, and determination of tumor displacement vectors after repeat manual tumor contouring. This combination of frameless wing board immobilization and free breathing scans are simplified set-up techniques with the potential to reduce time of treatment and improve patient comfort.

\section{Factors influencing positional accuracy}

Previously noted predictors of larger interfraction and intrafraction positional variability included type of immobilization device $[9,11]$, motion amplitude, [11] and increased treatment time [18], but these have not previously been evaluated in relation to frameless, freebreathing patient set-up. A statistically significant increase in tumor displacement on treatment СBCT was observed when tumor motion amplitude was $>9 \mathrm{~mm}$ on planning 4DCT. In agreement with Shah et al. [11], who demonstrated that upon multivariate analysis only treatment time, immobilization device, and respiratory excursion vector were significant predictors of variation of tumor position greater than $2 \mathrm{~mm}$. The effect of treatment time was not further evaluated in this analysis, since only small inter-patient variations in treatment time were observed reducing the likelihood to identify a significant effect on patient displacements.

\section{Volume changes during SBRT}

Few reports have evaluated volume change in SBRT with the use of CBCT. After observing substantial interfraction shifts during a 3-week delivery of SBRT that correlated with tumor volume reduction, Underberg et al. [19] concluded that repeat imaging must be performed during SBRT delivery. In contrast, utilizing a limited number of 4DCTs rather than evaluating inter/intrafraction CBCTs, Haasbeek et al. [20] noted volume change did not have relevant dosimetric consequence necessitating adaptive planning. Since initial 4DCTs obtained prior to the first fraction were used as a reference in Haasbeek et al. [20] study, the irrelevance of volume change on dosimetry may be partially explained by interval tumor growth. They noticed interval growth in $25 \%$ of patients. Our study utilized the GTV from initial CBCT of the first fraction as reference which eliminated the possibility of tumor growth as cause for tumor volume changes. In a more recent study, Galerani et al. [6] observed volume reduction of less than $14 \%$ in a majority of reviewed patients and assumed volume change was negligible. While similar average volume change, we noted that an average change greater than $12 \%$ over a course of treatment was significantly associated with increased interfractional tumor displacement. The effect of volume reduction on adaptive re-planning 
Citation: Schuster J, Myers M, Rosu M, Mukhopadhyay N, Weiss E (2013) Variations of the Tumor Position in Frameless Lung Sbrt: Assessment of Predictive Factors Including Tumor Volume Changes. J Nucl Med Radiat Ther 4: 147. doi:10.4172/2155-9619.1000147

Page 5 of 5

requires further analysis, especially in situations where normal tissue sparing is a concern, such as tumor positions close to central airways, brachial plexus or chest wall.

\section{Shortcomings}

Manual contouring is subject to variability that might affect this analysis. To limit concern that volume change was an artifact of contouring variation and to avoid interobserver variability, one physician completed all contours. Intraobserver variation was found to be small, and an independent verification of all contours was completed by a second physician.

Imaging blurring of CBCTs caused by respiratory motion and time-averaged voxels is a known limitation to accurately define the true tumor volume. As noted previously by Redmond et al. [21] respiratory motion does not demonstrate significant differences between simulation and treatment, therefore respiratory variations compared to initial CBCT should have limited affect on volume change. However, imaging factors and small sample size may have contributed to interfraction positional variation showing significant correlations only in ML direction which usually is affected least by respiratory motion.

\section{Conclusions}

Positioning accuracy of frameless immobilization with free breathing scans is comparable to more elaborate positioning techniques. While interfractional variations are often correctable with pretreatment $\mathrm{CBCT}$ tumor-based alignment, the frequency and timing of repeated intrafraction CBCTs is still investigational and is of particular interest in frameless SBRT and patients with higher risk tumor characteristics where increased positioning uncertainty is assumed such as tumors with large volume change, greater respiratory motion amplitude, and peripheral location. This study demonstrated that the likelihood of $>3 \mathrm{~mm}$ intrafraction displacements is low even with frameless set up reducing the need for reassessment of the tumor position during a fraction. Although limited by small patient numbers, the observed association of tumor volume changes with interfractional displacement may impact SBRT clinical delivery and warrants further study with consideration of adaptive planning.

\section{Acknowledgement}

This work has been partially supported by NIH grants P01CA116602 and P30CA016059.

\section{References}

1. Timmerman R, Paulus R, Galvin J, Michalski J, Straube W, et al. (2010) Stereotactic body radiation therapy for inoperable early stage lung cancer. JAMA 303: 1070-1076.

2. Onishi $H$, Shirato $H$, Nagata $Y$, Hiraoka M, Fujino M, et al. (2007) Hypofractionated stereotactic radiotherapy (HypoFXSRT) for stage I nonsmall cell lung cancer: updated results of 257 patients in a Japanese multiinstitutional study. J Thorac Oncol 2: S94-100.

3. Uematsu M, Shioda A, Suda A, Fukui T, Ozeki Y, et al. (2001) Computed tomography-guided frameless stereotactic radiotherapy for stage I non-small cell lung cancer: a 5-year experience. Int J Radiat Oncol Biol Phys 51: 666-670.

4. Martini N, Bains MS, Burt ME, Zakowski MF, McCormack P, et al. (1995)
Incidence of local recurrence and second primary tumors in resected stage lung cancer. J Thorac Cardiovasc Surg 109: 120-129.

5. Mageras GS, Pevsner A, Yorke ED, Rosenzweig KE, Ford EC, et al. (2004) Measurement of lung tumor motion using respiration-correlated CT. Int J Radiat Oncol Biol Phys 60: 933-941.

6. Galerani AP, Grills I, Hugo G, Kestin L, Mohammed N, et al. (2010) Dosimetric impact of online correction via cone-beam CT-based image guidance for stereotactic lung radiotherapy. Int J Radiat Oncol Biol Phys 78: 1571-1578.

7. Jaffray DA, Siewerdsen JH, Wong JW, Martinez AA (2002) Flat-panel conebeam computed tomography for image-guided radiation therapy. Int J Radiat Oncol Biol Phys 53: 1337-1349.

8. Létourneau D, Wong JW, Oldham M, Gulam M, Watt L, et al. (2005) Conebeam-CT guided radiation therapy: technical implementation. Radiother Oncol 75: 279-286.

9. Bissonnette JP, Franks KN, Purdie TG, Moseley DJ, Sonke JJ, et al. (2009) Quantifying interfraction and intrafraction tumor motion in lung stereotactic body radiotherapy using respiration-correlated cone beam computed tomography. Int J Radiat Oncol Biol Phys 75: 688-695.

10. Jensen HR, Hansen O, Hjelm-Hansen M, Brink C (2008) Inter- and intrafractional movement of the tumour in extracranial stereotactic radiotherapy of NSCLC. Acta Oncol 47: 1432-1437.

11. Shah C, Grills IS, Kestin LL, McGrath S, Ye H et al. (2012) Intrafraction variation of mean tumor position during image-guided hypofractionated stereotactic body radiotherapy for lung cancer. Int J Radiat Oncol Biol Phys 82: 1636-1641.

12. Guckenberger M, Meyer J, Wilbert J, Richter A, Baier K, et al. (2007) Intrafractional uncertainties in cone-beam CT based image-guided radiotherapy (IGRT) of pulmonary tumors. Radiother Oncol 83: 57-64.

13. Cho BC, van Herk M, Mijnheer BJ, Bartelink H (2002) The effect of set-up uncertainties, contour changes, and tissue inhomogeneities on target dosevolume histograms. Med Phys 29: 2305-2318.

14. Britton KR, Starkschall G, Tucker SL, Pan T, Nelson C, et al. (2007) Assessment of gross tumor volume regression and motion changes during radiotherapy for non-small-cell lung cancer as measured by four-dimensional computed tomography. Int J Radiat Oncol Biol Phys 68: 1036-1046.

15. Knap MM, Hoffmann L, Nordsmark M, Vestergaard A (2010) Daily cone-beam computed tomography used to determine tumour shrinkage and localisation in lung cancer patients. Acta Oncol 49: 1077-1084

16. Ikushima H, Balter P, Komaki R, Hunjun S, Bucci MK, et al. (2011) Daily alignment results of in-room computed tomography-guided stereotactic body radiation therapy for lung cancer. Int J Radiat Oncol Biol Phys 79: 473-480.

17. Sonke JJ, Rossi M, Wolthaus J, van Herk M, Damen E, et al. (2009) Frameless stereotactic body radiotherapy for lung cancer using four-dimensional cone beam CT guidance. Int J Radiat Oncol Biol Phys 74: 567-574.

18. Purdie TG, Bissonnette JP, Franks K, Bezjak A, Payne D, et al. (2007) Conebeam computed tomography for on-line image guidance of lung stereotactic radiotherapy: localization, verification, and intrafraction tumor position. Int J Radiat Oncol Biol Phys 68: 243-252.

19. Underberg RW, Lagerwaard FJ, van Tinteren H, Cuijpers JP, Slotman BJ, et al (2006) Time trends in target volumes for stage I non-small-cell lung cancer after stereotactic radiotherapy. Int J Radiat Oncol Biol Phys 64: 1221-1228.

20. Haasbeek CJ, Lagerwaard FJ, Cuijpers JP, Slotman BJ, Senan S (2007) Is adaptive treatment planning required for stereotactic radiotherapy of stage non-small-cell lung cancer? Int J Radiat Oncol Biol Phys 67: 1370-1374

21. Redmond KJ, Song DY, Fox JL, Zhou J, Rosenzweig CN, et al. (2009) Respiratory motion changes of lung tumors over the course of radiation therapy based on respiration-correlated four-dimensional computed tomography scans. Int J Radiat Oncol Biol Phys 75: 1605-1612. 